\title{
Physical parameters activating electrical signal distortions in polluted soils
}

\author{
Gabriella Losito and Rossana Angelini \\ Dipartimento di Ingegneria Civile, Università di Firenze, Italy
}

\begin{abstract}
Laboratory investigations and field measurements show that the electrical behaviour of polluted soils is strongly non-linear at low frequencies. This phenomenon can be related to the class and amount of pollutants. To measure this non-linearity, we used only monochromatic voltage waveform as input signal and analysed the current signals at first by means of the classical spectral analysis. In particular, the Total Harmonic Distortion \% (THD\%) and the Harmonic Distortion \%(HD\%) measure the non-linearity level and identify the frequency interval where the nonlinear electrical behaviour is activated. This frequency interval can be related to the pollutant molecular size. Open interpretative problems were the following: 1) phase localization of the signal deformation; 2) «local» amplitude of the applied signal activating the distortion, and 3) numerical fit of the distortion. We employed the wavelet analysis to study the phenomenon. The wavelet technique breaks up a signal into shifted and scaled versions of the original wavelet, which is a waveform of limited duration. These features of the wavelets allow us to obtain current components that can be interpreted on the bases of a real physical meaning. By using the wavelet analysis, we obtained the phase localization of the «oscillations» of the details and consequently the phase and amplitude of the applied signal. The sum of nine details provides a good numerical fit of the distorted signal. Starting from the wavelet analysis, we determined the physical conditions activating each distortion, testing some parameters on experimental data. The parameters that resulted most significant are the phase $\varphi$ of the distortion activation and the product $\mathrm{V}_{\text {in }} \Delta t(\mathrm{Vs})$ (where $\Delta t$ is the time interval corresponding to the said $\varphi$ and $\mathrm{V}_{\text {in }}$ is the integral tension applied to the sample on $\Delta t$ ). The latter parameter is in a very good agreement with field data of Advanced Monochromatic Spectral Induced Polarization (AMSIP) and restricts the physical interpretative hypotheses of distorted signals that are measured in the field. Typical experimental results will be shown as examples.
\end{abstract}

Key words induced polarization - wavelet decomposition - non-linear electrical behaviour electrical physical parameters

\section{Introduction}

This paper concerns the analysis of the nonlinear electrical behaviour of soils, especially polluted soils, at very low frequencies $(f \leq 0.1$

Mailing address: Dr. Gabriella Losito, Dipartimento di Ingegneria Civile, Università di Firenze, Via S. Marta 3, 50149 Firenze, Italy; e-mail: Losito@ dicea.unifi.it
$\mathrm{Hz}$ ). In fact, at these frequencies, the electrical response of a water saturated porous rock is sensitive to the properties of the mineral-water interface and to the polluted particles floating inside the fluid. This fluid/solid interaction produces a non-linear electrical behaviour and consequently distortions of the output signals: if pure harmonic signals are used as input function, the frequency content of the output signals is not monochromatic (Olhoeft, 1979). Currently rocks and soils are characterised in the field by their DC resistivity $\rho(\Omega \mathrm{m})$, but in the laboratory also by the complex electrical resistivity. If the phenomena are linear, the frequency dispersion of $\rho$ can correctly de- 
scribe the electrical polarizations; the standard Induced Polarization (IP) can be employed (both Frequency Domain FD and Time Domain TD) and the classical Cole-Cole (1941) model can be used in the interpretation of electronic and ionic polarizations (Patella, 1993). In the case of non-linear electrical behaviour, the electrical characterization of the medium must be completed by other parameters, both should be obtainable in the field and in the laboratory (Finzi-Contini, 1971). Recent applications of Advanced Monochromatic Spectral Induced Polarization (AMSIP), a technique based on the use of sinusoidal waveforms as input signals (Losito et al., 1998), provides a quantitative evaluation of soil pollution by means of the relative Total Harmonic Distortion \% (THD\%). This parameter is computed from the Fourier spectral analysis applied to the output signals. Here we must note that this parameter represents only a global measure of the non-linearity produced by the presence of pollutants at the mineral-water contact. It cannot discriminate the physical conditions activating the waveform distortions specific for each pollutant.

To solve this problem, we present in this paper an application of the wavelet analysis to analyse the non-linear components of the output signals produced by the electrochemical interaction between some pollutants and silica sands. The wavelet analysis was selected, because this technique is based on functions that are time limited and amplitude modulated (Grossman and Morlet, 1984; Holschneider, 1995) and defines the physical conditions (like time, phase and applied voltage) activating local perturbations. The research criteria and application results are described in Section 4.1.

\section{Experimental studies on polluted samples}

The electrical behaviour of artificial and natural samples has been studied in laboratory as a function of frequency $(0.002 \mathrm{~Hz}-100 \mathrm{kHz})$ and Voltage Amplitude (0.5-5 V), using the 4-electrode array technique (Losito, 1989). Hydrated artificial samples are: 1) a pure watersaturated silica sand $\left.\left(\mathrm{Si}-\mathrm{H}_{2} \mathrm{O}\right) ; 2\right) \mathrm{Si}-\mathrm{H}_{2} \mathrm{O}$ polluted by, respectively, motor-oil, trichloroethylene, dermo-nitrogen $\left(\mathrm{Si}-\mathrm{H}_{2} \mathrm{O}+\mathrm{Dn}\right)$, stomp
$\left(\mathrm{Si}-\mathrm{H}_{2} \mathrm{O}+\mathrm{Stomp}\right)$ and nitrophoska gold (the last three are fertilisers); 3) a pure watersaturated calcite sand; 4) pure water-saturated calcite-silica sand polluted by, respectively, motor-oil and trichloro-ethylene. Natural samples have been studied after water saturation and are: 1) soil fertilised by dermo-nitrogen (Soil + Dn); 2) natural soil (Nat Soil); 3) soils of dye-works; 4) soils and percolates of a waste deposit area (Dondoli et al., 1994; Losito et al., 1995, 1998).

The stomp is a fertiliser that uses, as active principle, Pendimethalin, an aniline derivative, while nitrophoska gold is an inorganic fertiliser.

We focused our attention on dermonitrogen, a fertiliser used in nurseries (organic nitrogen with Organic N: $11 \%$, Organic biological C: $40 \%$; Cr: $3 \%$ ). We must outline its high content of $\mathrm{Cr}$ that is a strong pollutant. An example of pollution effect on electrical signals is shown in fig. 1. This figure shows the comparison between the non-linear component of the current density (output signal) of pure silica sand and silica sand polluted by dermo-nitrogen. Measurements were performed at $0.002 \mathrm{~Hz}$. We can observe that dermo-nitrogen produces higher distortion in the electrical signals than pure silica sand.

Laboratory investigations were carried out on the bases of the following experimental criteria:

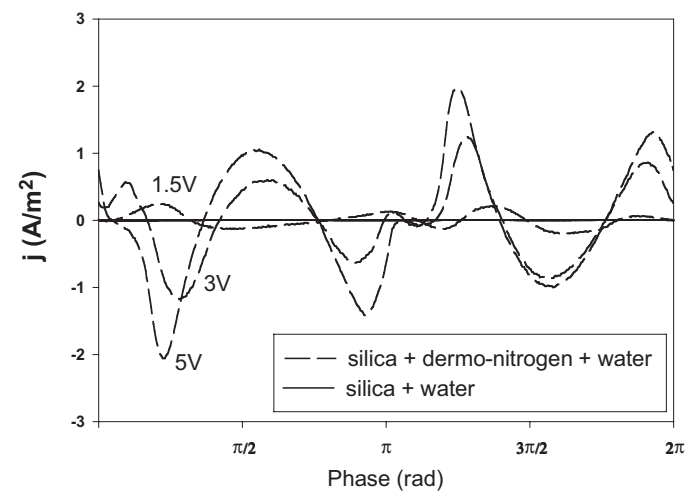

Fig. 1. Non-linear component of current density signals $j\left(\mathrm{~A} / \mathrm{m}^{2}\right)$, as function of phase $(\mathrm{rad})$ : pure silica sand (solid line) and silica sand polluted by dermo-nitrogen (dashed line). The working frequency is $0.002 \mathrm{~Hz}$. 

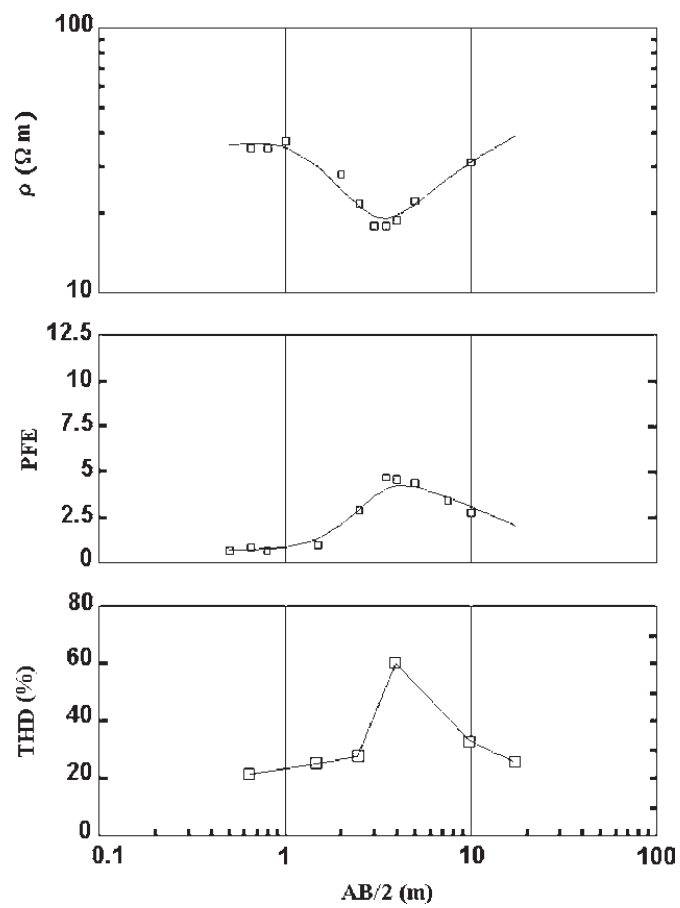

Fig. 2. Electrical responses of a polluted soil with dermo-nitrogen: resistivity $\rho[\Omega \mathrm{m}]$, Percent Frequency Effect (PFE) and Total Harmonic Distortion \% (THD\%) of Advanced Monochromatic Spectral Induced Polarization (AMSIP). Note the strong trend correlation if the energizing half-distance $\mathrm{AB} / 2$ is around $4 \mathrm{~m}$.

1) only pure sinusoidal waveforms of the applied electrical potential were used; 2) the electrical parameters were calculated in the frequency range $0.002 \mathrm{~Hz}-100 \mathrm{kHz} ; 3$ ) the electrical signals of samples were analysed by means of the Fourier Spectral Analysis, to obtain a measure of the distortion phenomena; 4) the differences between the normalised input and output signals have been calculated to locate the critical phases and voltages that activate the signal distortions; 5) the sample electrical behaviour was interpreted on the bases of chemical analyses.

For all samples the following electrical parameters were calculated: complex electrical resistivity $\rho(\Omega \mathrm{m})$; loss tangent $\tan \delta$; Total Harmonic Distortion THD\% $(\omega)$ and Harmonic
Distortion HD $\%(\omega)$ of the output electrical signals.

Joining the classical electrical parameters to THD\%, it is possible: 1) to identify the polarization phenomena; 2) to know the class of polarization on the bases of the frequency range; 3 ) to evaluate the polarization distortion size using the THD\% parameter.

To evaluate the soil electrical behaviour in the field we used a combined procedure. The classical electrical methods Vertical Electrical Sounding (VES) and Induced Polarization (IP) were combined with the new technique of Advanced Monochromatic Spectral Induced Polarization (AMSIP). Figure 2 shows field results related to an application of this combined technique to a soil polluted by dermo-nitrogen. The strong correlation of $\rho(\Omega \mathrm{m})$, Percent Frequency Effect (PFE) and THD\% highest variations is evident if the energizing halfdistance $(A B / 2)$ is around $4 \mathrm{~m}$. This behaviour can be attributed to a dermo-nitrogen level that is a high polarizable material.

\section{The wavelet analysis}

Application of Fast Fourier Transform leaves various questions open. We tried to solve these questions using the wavelet analysis in order to: 1) study phase localization of deformation signals; 2) identify physical conditions that activate each distortion; 3 ) fit distorted signals, and 4) find a relation between a single component and a physical phenomenon.

The non-linear component of rock electrical behaviour has been represented by the relative residuum between the output signal and its fundamental harmonic. We analysed relative residua of density current signals because the current and voltage output signals have a similar waveform, and, as is well known, the current flow inside the sample is the physical phenomenon induced by the voltage application (Loeb, 1976). The percent relative residua of current density $j$ $\left(\mathrm{A} / \mathrm{m}^{2}\right)$ give the phase and the frequency localization of distortion peaks (see fig. 3a,b concerning hydrated silica sand polluted by dermo-nitrogen) but the physical simulation of distortion remains an open problem. Recently, 

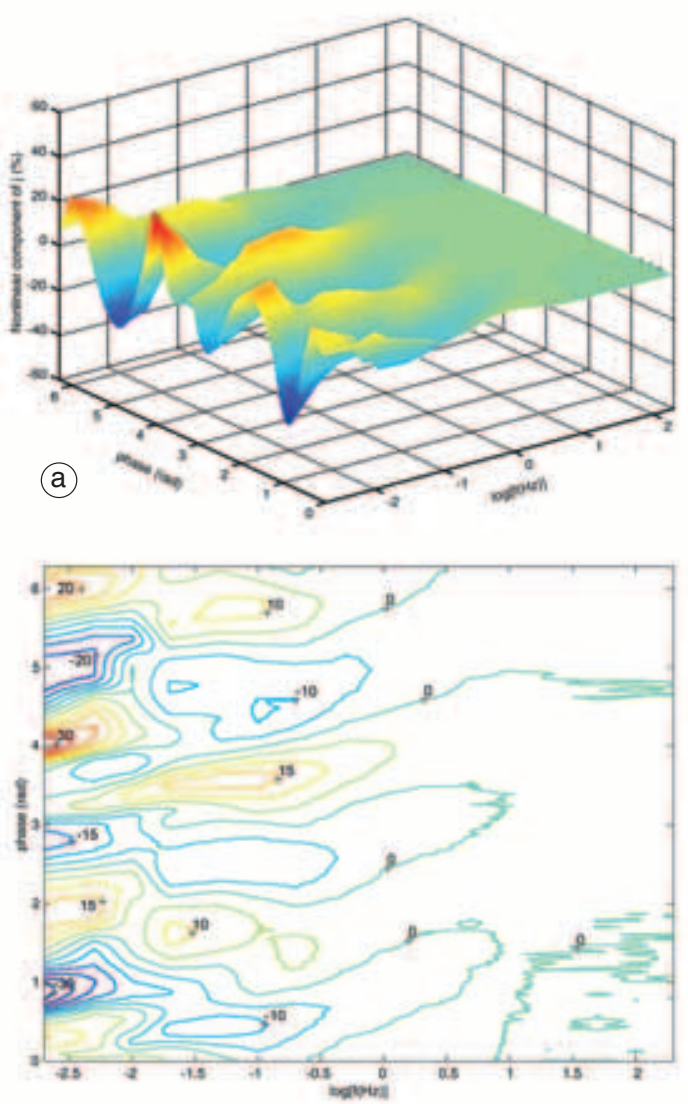

(b)

Fig. 3a,b. Percent non-linear component of $j$ signals, concerning a silica sand polluted by dermo-nitrogen, as frequency $(0.002 \mathrm{~Hz}-200 \mathrm{~Hz})$ and phase $(\mathrm{rad})$ function. The applied voltage is $3 \mathrm{~V}$. a) $3 \mathrm{D}$ representation and b) the corresponding contour level representation.

to quantify the electrical signal distortions produced by pollutants/mineral interactions, and to define the physical conditions activating the non-linearities, we used the wavelet analysis.

For the background theory relative to the use of wavelet analysis see Daubechies (1992), Coifman et al. (1992), Meyer (1990), Wicherhauser (1994). The signal wavelet analysis has been carried out on the following steps: 1) sample selection and evaluation of the distortion of $j$ signals; 2) selection of a wavelet function; 3) wavelet decomposition of relative residua;
4) determination of physical conditions activating distortions.

Here we recall the mathematical definition of the Continuous Wavelet Transform (CWT) of a temporal signal $x(t)$

$$
\begin{gathered}
\text { CWT }[x(t)]=C(a, b)= \\
\text { I }=\frac{1}{\sqrt{a}} \int_{-\infty}^{\infty} x(t) \bar{\Psi}\left(\frac{t-b}{a}\right) d t
\end{gathered}
$$

where $t$ (s) is the time, $\overline{\Psi(t)}$ is the complex conjugate function of the mother wavelet (timelimited, made of phase and frequency modulated oscillations), $b$ (s) is the time translation factor; $a$ is a dimensionless scale factor $(a<1, \psi$ compressed, $a>1, \psi$ expanded). Using the (3.1) we can calculate the $C(a, b)$ coefficients, that are a correlation index between the wavelet mother translated by $b$ and scaled by $a$, and the signal part under examination: high values of $C(a, b)$ correspond to a high correlation. It is important to point out that the adjective «continuous» is referred not to $t$, but to $a$ and $b$. In fact, the Discrete Wavelet Transform (DWT) is the CWT with discrete values for $a$ and $b$

$$
\begin{aligned}
& C(j, n)=\int x(t) \bar{\Psi}_{j n}(t) d t= \\
= & 2^{-j / 2} \int x(t) \bar{\Psi}\left(2^{-j} t-n\right) d t
\end{aligned}
$$

where $a=2^{j}$ and $b=n a=n 2^{j}$.

We selected a multiple-level wavelet decomposition technique (Mallat, 1989) of $\boldsymbol{j}$ relative residua, with nine levels, according the following steps. First the signal is decomposed in approximation A1 (its low frequency component) and in detail D1 (its high frequency component). The process is iterative (fig. 4a) and produces linked approximations and details

$$
\begin{gathered}
D_{j}(t)=\sum_{n} C(j, n) \Psi_{j, n}(t), \\
A_{j}(t)=\sum_{j>J} D_{j}(t) .
\end{gathered}
$$



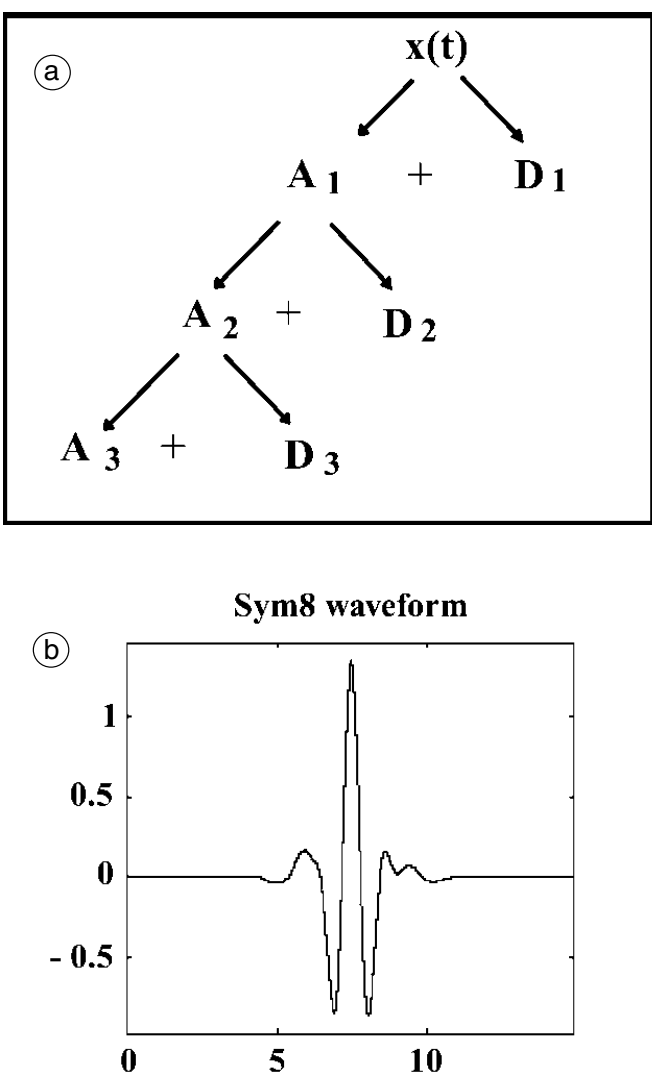

Fig. 4a,b. a) Scheme of iterative procedure for multiplelevel wavelet decomposition. b) Waveform of the selected wavelet: sym8.

Several wavelet functions were tested. In our selection, we followed the following basic criteria: the details must help in the physical description of the phenomenon and a particular detail can simulate a non-linear polarization. Finally we chose the mother wavelet sym8 (for its mathematical description see Daubechies, 1992); its form is shown in fig. 4 b.

Some basic observations about Fourier and wavelet analysis are interesting. The Fourier analysis is based on sinusoidal waves (so signal infinitely repeated in time and regular waveform) and so it decomposes a signal into sinusoidal waves. The wavelet analysis instead is based on mother wavelet $\psi(t)$ (so time-limited and free waveform) and it decomposes a signal into translated and scaled $\psi(t)$ functions: so wavelet analysis is more suitable for decomposition of signals with low frequency component and large local changes. In fact, using lower scale factors $(a<1)$ we obtain compressed wavelets with a high time resolution. They can be useful in the simulation of a short signal part and its high frequency behaviour using low-level details. Instead, using higher scale factors $(a>1)$ we use expanded wavelets with a minor time resolution. They can be compared with a long signal part to identify its low frequency components (i.e. its trend characterised by high level details). As regards the numerical simulation of local deformations, in Fourier analysis they are periodic elements. In fact it hypothesises that the local deformations repeat themselves during the whole signal and this is obviously a not realistic situation in non-linear behaviour. The wavelet analysis instead is able to point out the single distortions and give their exact time localization, without their repetition throughout the whole signal, as in the Fourier technique. So we can conclude that wavelet analysis components can be correlated with the physical parameters that produce the non-linear polarization of the samples.

Fitting examples calculated using the two methods are shown in fig. 5a-c. We can observe the higher fitting quality that is obtained by the wavelet method (W9, dash-dotted line). This fitting has been computed summing all nine details while Fourier fitting (F, dashed line) is computed with the contribution of harmonics with percent amplitude not below $0.7 \%$ of the principal one.

\section{Application of wavelet analysis to electrical signals of polluted samples}

We performed a wavelet decomposition with nine levels of all residual signals at $0.002 \mathrm{~Hz}$ using the sym8 function and we found that the details from 5th to 8th level are the most interesting and the largest components, that can describe polarization. In fact, more low-level details (D1, D2, D3, D4) represent principally noise, while D9 and A9 represent the low frequency components (trend). 

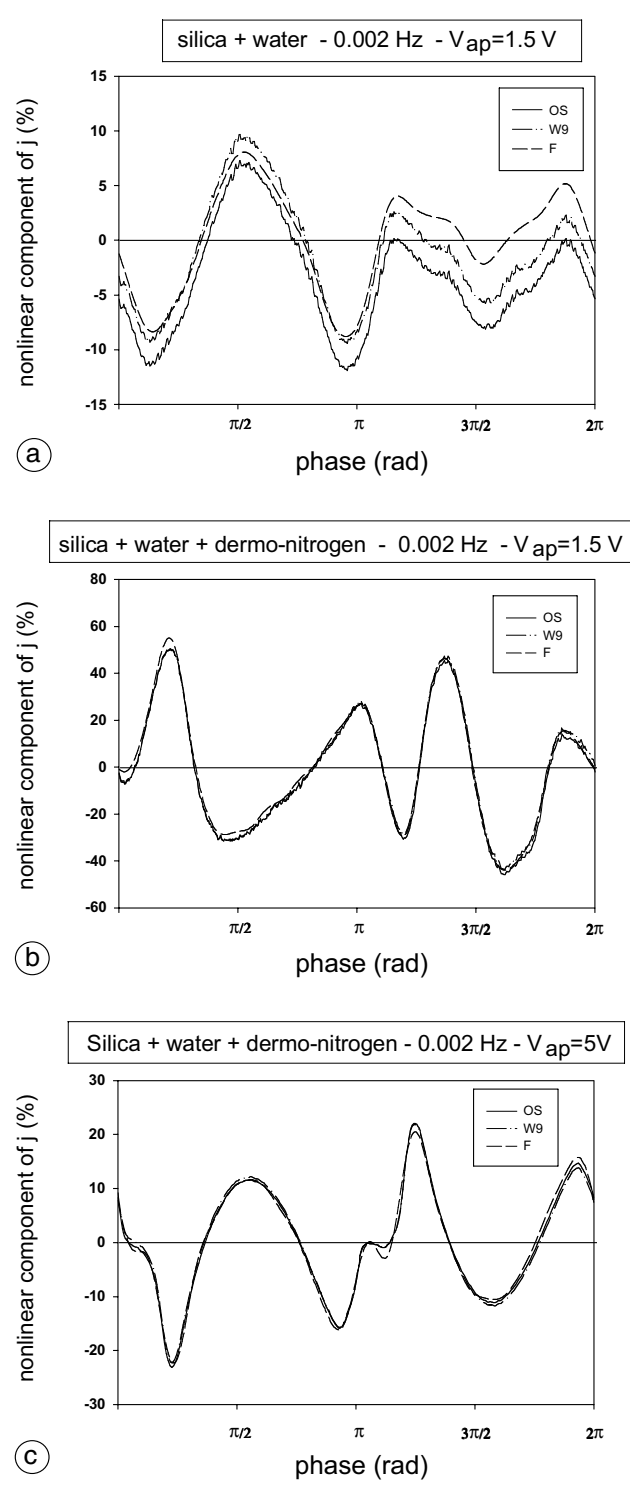

Fig. 5a-c. Comparison between relative residua signals (OS, solid) as phase (rad) function and its fitting obtained by using Fourier analysis (F, dashed) and wavelet analysis (W9, dash-dotted): a) pure silica sand with $\mathrm{V}_{\mathrm{ap}}=1.5 \mathrm{~V}$; b) silica sand polluted by dermonitrogen with $\mathrm{V}_{\mathrm{ap}}=1.5 \mathrm{~V}$; c) the same sample with $\mathrm{V}_{\mathrm{ap}}=5 \mathrm{~V}$. All the examples are obtained at a working frequency of $0.002 \mathrm{~Hz}$. The wavelet fitting is obtained summing all the 9 calculated details. The Fourier fitting is obtained summing the harmonic components of relative amplitude $A \geq 0.7 \%$.
Then, we made a final selection of the samples to study their behaviour in detail. First, we chose samples with very high non-linear behaviour (silica polluted by dermo-nitrogen and stomp). Then, we chose fertilised soil to make a comparison between natural and artificial samples with the same pollutant. Finally, we chose silica sand and natural soil similar to the fertilised one as calibration samples.

We made a wavelet decomposition of all relative residua $>5 \%$ for the five selected samples. The computed details allow us to localize the perturbation phase and to state a relationship between detail waveforms and applied signal waveforms. In fact, it is possible to relate the presence of the most important peaks to the sign of energizing signal and its derivative. A common result for all the studied samples is that, for all the details (except D8) there are high distortions at the change of applied signal sign. This is a proof that the two semi-waves (positive and negative) introduce different deformations, as confirmed also by the asymmetry of the relative residuum. In fig. 6 we can see the decomposition results relative to silica sand polluted by dermo-nitrogen, with applied tension respectively of $1.5 \mathrm{~V}$ and $3 \mathrm{~V}$. D8 is more influenced by the increment of applied tension, but in general, for all the levels, the peak presence in phase depends on applied tension values (see for example D5).

\subsection{Physical parameters activating the non- linearity}

To find physical parameters activating the non-linearity, we focused our attention on positive peaks near $\pi$ for the three first details and on the maximum positive peak for D8, trying to define the physical conditions activating this particular deformation. We selected the following parameters:

1) phase $\varphi$ of distortion start (rad);

2) energizing time $\Delta t(\mathrm{~s})$;

3) integral tension $\mathrm{V}_{\mathrm{in}}=\int_{0}^{\varphi}\left|\mathrm{V}_{\mathrm{AP}} d \varphi\right|(\mathrm{V})$;

4) the product $V_{\text {in }} \Delta t(\mathrm{Vs})^{0}$. 

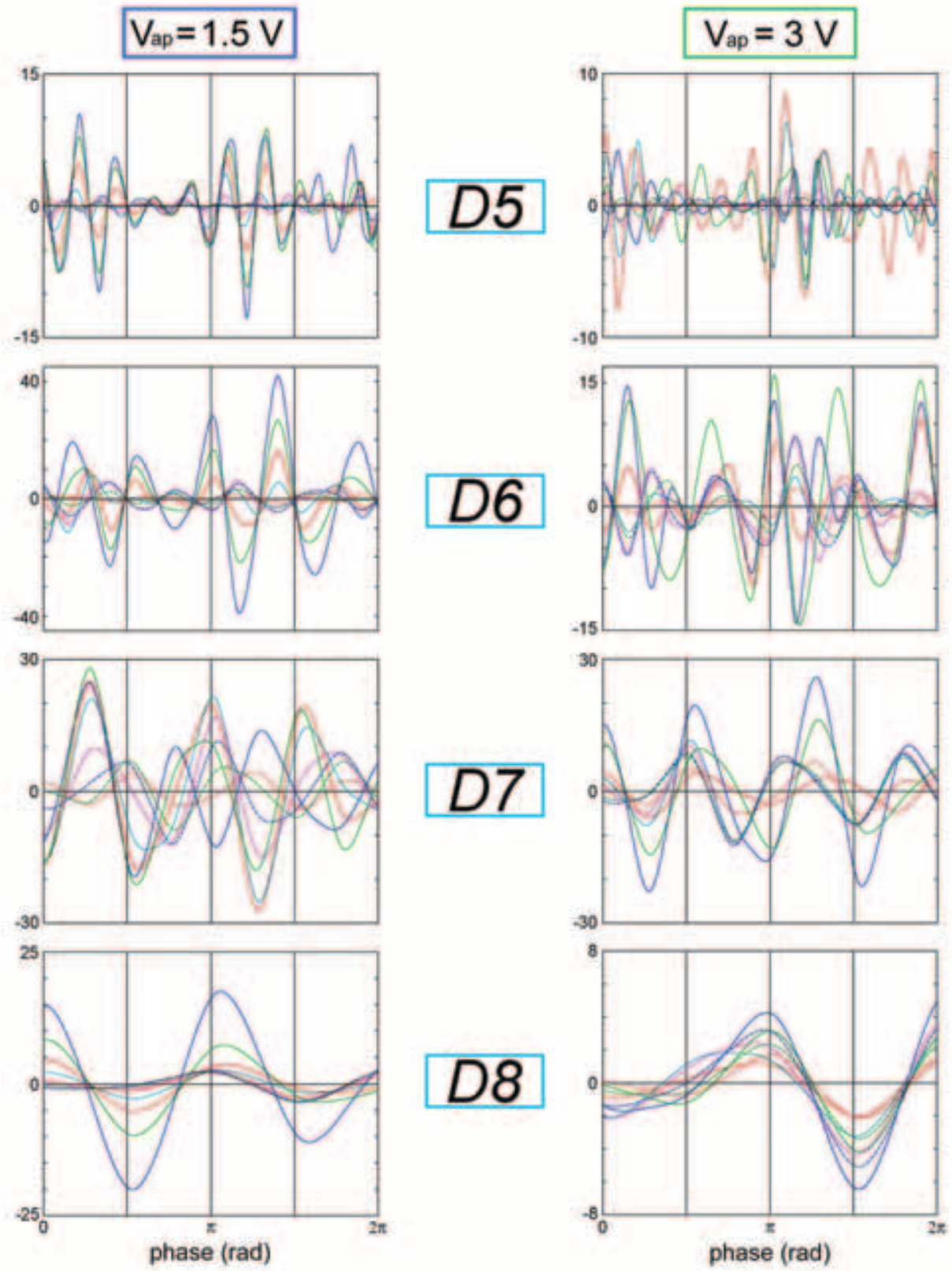

$$
f(\mathrm{~Hz})-0.002-0.005-0.01-0.02-0.05-0.1-0.2-0.5
$$

Fig. 6. Wavelet analysis of the $j$ signals \% non-linear component. Details D5-D8 concerning a silica sand polluted by dermo-nitrogen. Applied voltage signals: left column $1.5 \mathrm{~V}$, right column $3 \mathrm{~V}$. Working frequencies as described in the legend. 

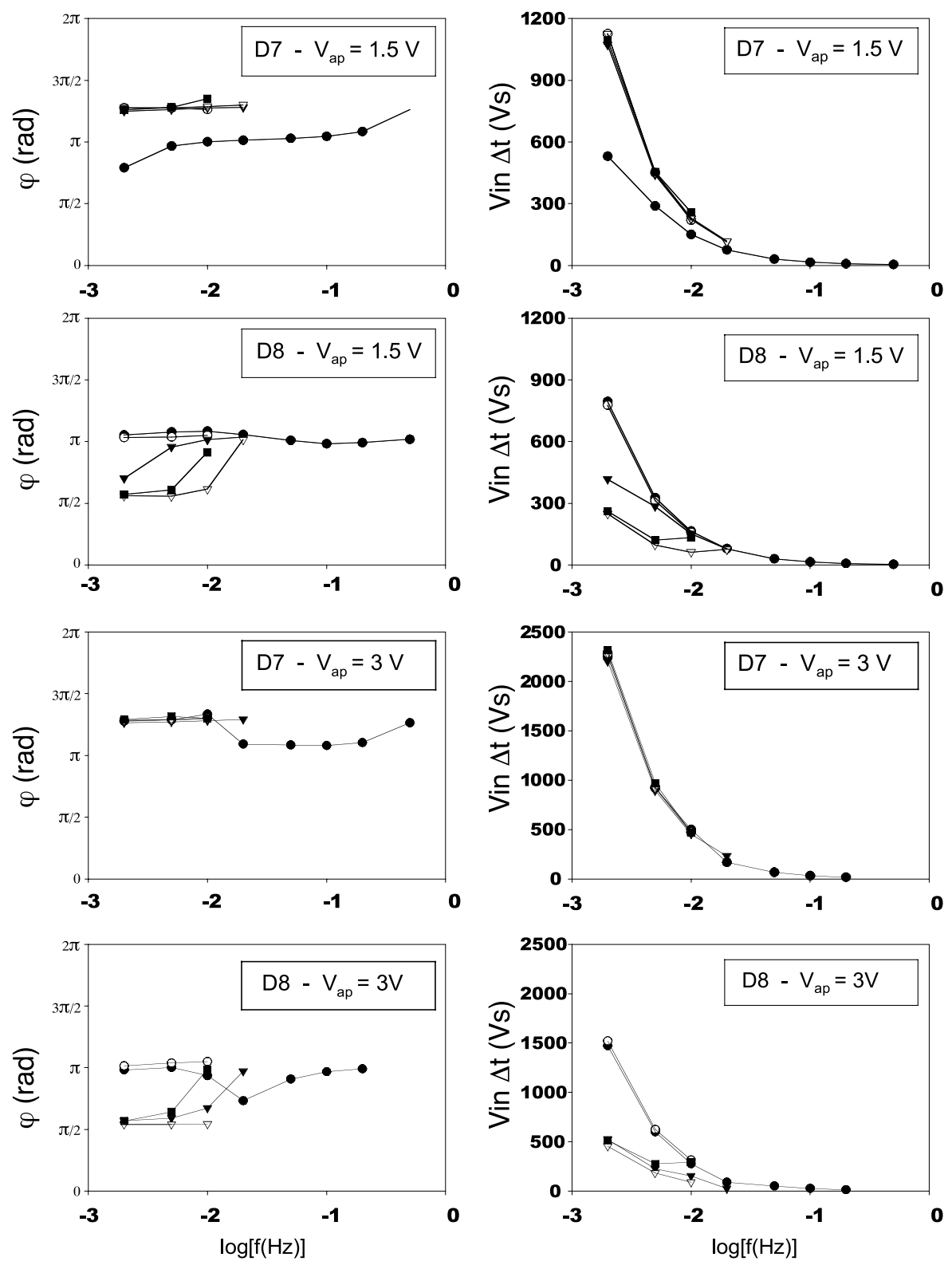

Si- $\mathrm{H}_{2} \mathrm{O}+\mathrm{Dn} \quad$ Soil + Fert $\quad \mathrm{Si}-\mathrm{H}_{2} \mathrm{O}+$ Stomp $\quad$ Si- $\mathrm{H}_{2} \mathrm{O} \quad$ Nat Soil

Fig. 7. Frequency trend of phase $\varphi$ and the parameter $\mathrm{V}_{\text {in }} \Delta t$ (see text) of the details $\mathrm{D} 7$ and $\mathrm{D} 8$ of the five selected samples. Applied tension $1.5 \mathrm{~V}$ (upper part of the figure) and $3 \mathrm{~V}$ (lower part of the figure). $\mathrm{Si}+\mathrm{Dn}$ : silica sand with dermo-nitrogen; Soil + Fert: soil fertilised by dermo-nitrogen; Si + Stomp: silica sand with stomp; Si + Water: pure silica sand; Nat Soil: natural soil. Observe that D8 level allows to discriminate the presence of pollutant. 
Physical parameters activating electrical signal distortions in polluted soils
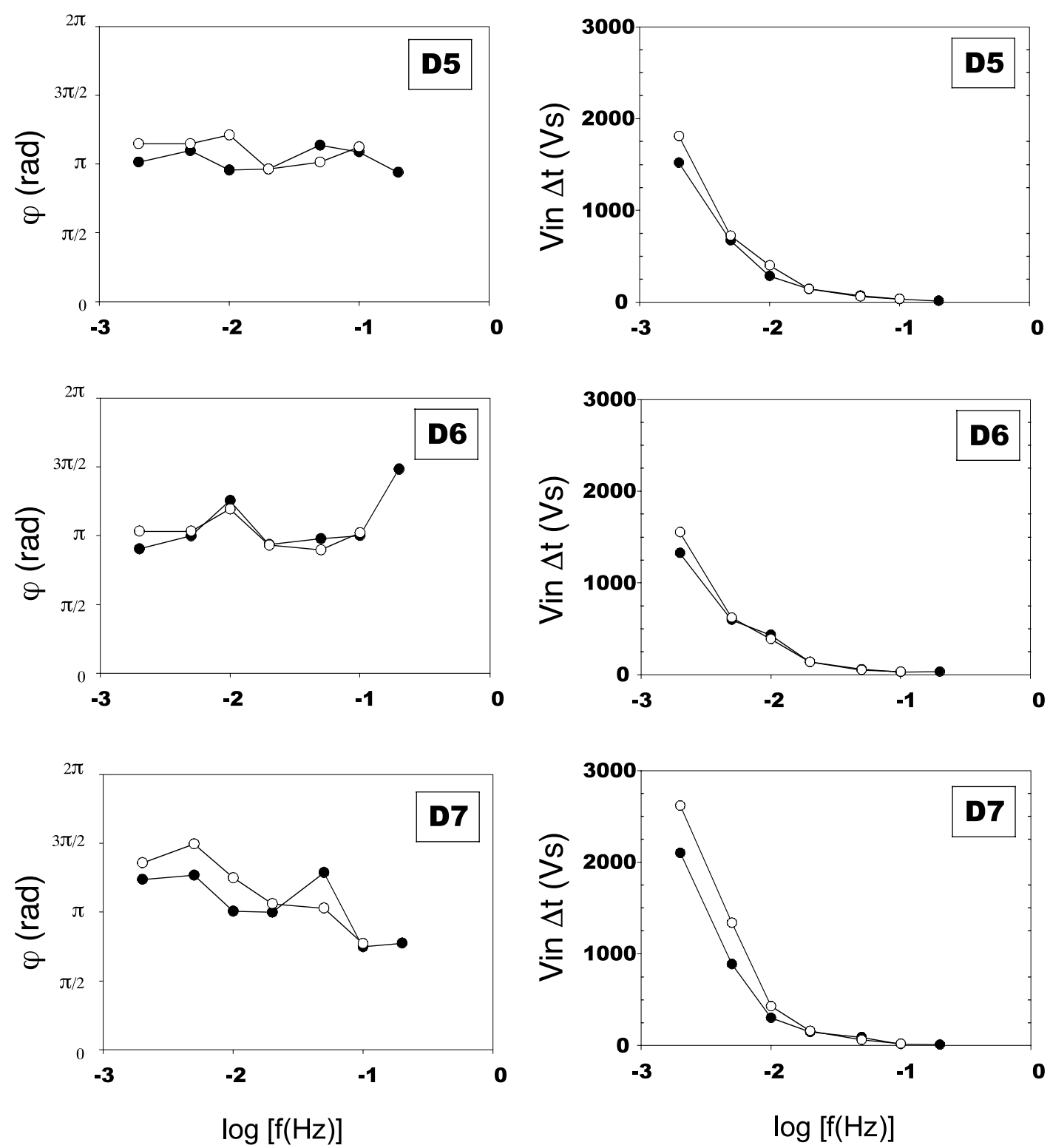

$$
\text { Vap signal : } \quad \longrightarrow \text { sine } \quad \longrightarrow \text { cosine }
$$

Fig. 8. Comparison between frequency trend of $\varphi$ and $\mathrm{V}_{\text {in }} \Delta t$ obtained by applying a cosine signal (white point) and a sine signal (black point) to silica sand polluted by trichloro-ethylene. The applied tension is $3 \mathrm{~V}$. Note the no effect applied signal phase. 
We must note that in the calculation of $\mathrm{V}_{\text {in }}$ (i.e. the total tension applied, at the working frequency, to the sample until the phase $\varphi$ ) we considered the absolute value of the applied signal because the provided tension during the negative semi-wave is a further energy contribution to the sample and not an energy subtraction from the energy provided during positive semi-wave. Then we selected the most interesting parameters: $\varphi$ and $\mathrm{V}_{\text {in }} \Delta t$. The frequency trends of $\varphi$ and $\mathrm{V}_{\text {in }} \Delta t$ are shown in fig. 7. We can see that for D7 and D8 at $1.5 \mathrm{~V}$ the $\mathrm{V}_{\text {in }} \Delta t$ parameter is able to discriminate the samples with dermo-nitrogen. It is interesting that for the two details we have an opposite situation. In fact, for D7 (more high frequency component) the distortions due to dermo-nitrogen presence are characterised by lower values of $\mathrm{V}_{\text {in }} \Delta t$ product compared to the distortions in the other samples. Instead, for D8 (more low frequency component) the maximum positive peak in the samples polluted by dermo-nitrogen is activated by major values of $\mathrm{V}_{\text {in }} \Delta t$ parameter compared to the peak in the other samples.

The low frequency component is probably influenced by polarization phenomena due to the largest molecules (for example organic ones) with a major electrical inertia. This means that, with the same applied signal frequency, the peak is present at higher values of $\varphi$ : consequently both $\Delta t$ and $\mathrm{V}_{\text {in }}$ parameters will assume higher values and so their product. Instead, the highest frequency component is probably influenced by polarization phenomena due to smaller molecules, with a minor electrical inertia, mobilised by minor values of $\mathrm{V}_{\text {in }} \Delta t$ product.

Only at low tension can D7 discriminate dermo-nitrogen while D8 well discriminates it at all tensions. So we can conclude that $1.5 \mathrm{~V}$ is the most suitable value of applied signal in pointing out the non-linear polarization phenomena, while with high values there is a saturation effect, experimentally evaluated (Marshall and Madden, 1959; Madden and Cantwell, 1967; Dondoli et al., 1994; Losito et al., 1995).

To verify $\varphi$ and $\mathrm{V}_{\text {in }} \Delta t$ we conducted the following experiment: we applied both sine (black point) and cosine (white point) waveform signals to an artificial polluted sample (silica sand with trichloro-ethylene). Also in this case these parameters were independent of applied signal phase and consequently independent of local applied tension value, as we can see in fig. 8 . This result indicates that the sample behaviour depends on applied tension history.

We think that the laboratory results can be extended to the interpretation of field data that show high non-linearities (see fig. 2 where the value of $60 \%$ for THD $\%$ depends on a soil level polluted by dermo-nitrogen). In fact, even if the field measurements are obtained at energizing frequencies (i.e. in the range 0.01-0.1 Hz), the output signals are acquired after a certain number of energizing cycles. In this way the total energizing time can be compared with the laboratory one obtained with minor working frequencies.

\section{Conclusions}

The principal aim of this work was to study the non-linearity of the electrical outputs produced by polarization phenomena in polluted rocks.

The application of the Advanced Monochromatic Spectral Induced Polarization (AMSIP) to laboratory samples represents a useful starting point. This technique allows to verify not only the presence of polarization phenomena, but it also yields a physical interpretation of the problem, on the basis of the polarization frequency range. In addition, the AMSIP technique gives a first «global» measure of the non-linearity entity, thanks to the Total Harmonic Distortion $\%$ parameter, obtained starting from the Fourier analysis.

By comparison with the Fourier analysis, the wavelet analysis allows a «local» study of the electrical signal deformations. This is due to the particular characteristics of the functions used in this type of analysis. In fact they are timelimited and made of phase and frequency modulated oscillations, and this makes them suitable to study signals with large local changes (i.e. high frequency component) put upon low frequency components.

An important goal is that wavelet analysis, instead of Fourier one, not only gives a good numerical fitting but also frequency components 
correlated with the real physical phenomenon. For Fourier analysis, the local deformations are periodic elements while wavelet analysis permits their exact time localization. The analysis of relative residua and their details gives a precise localization in phase of the single distortions and how they develop throughout the output signal.

The distortion localization in phase $\varphi(\mathrm{rad})$ seems to give a way to determine the physical conditions for deformation activation. In fact, knowing the applied signal frequency, it is possible to evaluate $\Delta t(\mathrm{~s})$, the energizing time necessary to activate a particular distortion. In addition, it is also possible to evaluate $\mathrm{V}_{\text {in }}(\mathrm{V})$, the total tension provided to the sample until $\varphi$.

However, the most interesting parameter to define the physical conditions of activation is the product $\mathrm{V}_{\text {in }} \Delta t(\mathrm{Vs})$. The reason for this is that the sample electrical behaviour depends not only on the given tension, but also on the energizing time (i.e. the time during which this tension is given to the sample). We can see that the product $\mathrm{V}_{\text {in }} \Delta t$ for D7 and D8 discriminates the samples with dermo-nitrogen.

\section{REFERENCES}

COIFMAN, R.R., Y. MEYER and M.V. WICKERHAUSER (1992): Wavelet analysis and signal processing, in Wavelets and their Applications, edited by M.B. RUSKAI et al. (Jones and Bartlett)

CoLE, K.S. and R.H. CoLE (1941): Dispersion and absorption in dieletrics - I: Alternating current characteristic, J. Chem. Phys., 9, 341-351.

DAUBECHIES, I. (1992): Ten Lectures on Wavelets (SIAM), pp. 225.

Dondoli, A., G. Losito and A. TrovA (1994): Possibilità del metodo elettrico nell'individuazione di inquinanti in acquiferi e terreni, Ing. Sanit. Amb., 3, 22-29.

FINZI-CONTINI, G. (1971): Theoretical study on membrane polarization and complex mobility in moist rocks, Geol. Surv. Can. Pap., 71-1, B, 53-65.
Grossman, A. and J. Morlet (1984): Decomposition of Hardy functions into square integrable wavelets of constant shape, SIAM J. Math. Anal., 15, 723-736.

HolschneIDER, M. (1995): Wavelets: an Analysis Tool (Clarendon Press, Oxford), pp. 324

LOEB, J. (1976): Experimental and Theoretical Aspects of Induced Polarization - Vol. 2: Macroscopic and Microscopic Theories (Gebrüder Borntraeger, Berlin Stuttgart), pp. 335.

Losito, G. (1989): A new rock physics laboratory apparatus with programmed thermo-baric cycles and controlled pore fluid internal pressure (theory, hardware-software, electrical measurements), CNR-ENEA, PFE SP En. Geot., LB-21, 1-150.

Losito, G. and A. TrovA (1999): Wavelet technique applied to localization and interpretation of electrical signal distortions produced by pollutants in soils, in 5th EEGSES Environmental and Engineering Geophysics, Budapest (Hungary) September 6-9, Ext. Abstract DE10.

Losito, G., M. MuschIETTI and A. TROVA (1995): Electrical effects of pollutants in earth soils and sedimentary rocks, in 1st Meeting of Environmental and Engineering Geophysics, 57-60.

Losito, G., A. Trova and I. D’Urso (1998): Frequency and DC electrical behaviour of polluted sediments in industrial areas: field and laboratory investigations, in EEGS Barcelona (Spagna), 14-17 September 1998, extended abstract, 35-38.

Madden, T.R. and T. CANTwell (1967): Induced Polarization. A review, Min. Geophys. (S.E.G.), II, 373-400.

Mallat, S. (1989): A theory for multiresolution signal decomposition: the wavelet representation, IEEE Trans. Pattern Analysis and Machine Intelligence, vol. 11 (7), 674-693.

Marshall, D.J. and T.R. MAdDEN (1959): Induced Polarization. A study of its causes, Geophysics, 24, 790-816.

MEYER, Y. (1990): Wavelets and Operators, translated by D.H. SALINGER (Cambridge Univ. Press, 1993), pp. 223.

Olhoeft, G.R. (1979): Nonlinear electrical properties, in Nonlinear Behaviour of Molecules, Atoms and Ions in Electric, Magnetic and Electromagnetic Fields, edited by L. NEEL (Elsevier), 395-409.

PATElla, D. (1993): I principi metodologici della magnetotellurica su mezzi generalmente dispersivi, Ann. Geofis., 36 (suppl. to n. 5-6), 147-160.

WICKERHAUSER, M.V. (1994): Adapted Wavelet Analysis from Theory to Software Algorithms (A.K. Peters), pp. 241. 